\title{
Photonic composite materials from cellulose nanorods and clay nanolayers
}

\author{
Ana C. Trindade ${ }^{1, a}$, Miguel Carreto ${ }^{2}$, Geir Helgesen ${ }^{3}$, Kenneth D. Knudsen ${ }^{3}$, \\ Florian Puchtler ${ }^{4}$, Josef Breu ${ }^{4}$, Susete Fernandes ${ }^{2}$, Maria Helena Godinho ${ }^{2}$, and \\ Jon Otto Fossum ${ }^{1}$ \\ ${ }^{1}$ Department of Physics, Norwegian University of Science and Technology, Trondheim, \\ Norway \\ 2 i3N/CENIMAT, Department of Materials Science, Faculty of Science and Technology, \\ Universidade NOVA de Lisboa, Campus de Caparica, Caparica, Portugal \\ ${ }^{3}$ Institute for Energy Technology, 2027 Kjeller, Norway \\ ${ }^{4}$ Lehrstuhl für Anorganische Chemie I, Universität Bayreuth, 95440 Bayreuth, Germany
}

Received 3 February 2020 / Accepted 27 July 2020

Published online 16 November 2020

\begin{abstract}
Cellulose nano crystals (CNCs) are promising materials for energy efficient buildings related to the control of reflectivity and heat absorption/reflection of light. In this sense it is important to improve CNCs films fire retardant properties, which can be achieved by adding clays. Cellulose nanocrystals (CNCs) and nanolayers obtained from Sodium Fluorohectorite (NaFh) synthetic clay are both known to form liquid crystalline phases in aqueous suspensions. CNCs form cholesteric phases, which structure is preserved after water evaporation, while dry $\mathrm{NaFh}$ nanolayers aligned films collapse. In this initial work, it is shown that CNCs are compatible with $\mathrm{NaFh}$ clay. We demonstrate that the liquid crystalline phase of CNCs in water is not destroyed by the presence of $\mathrm{NaFh}$ nanolayers. The NaFh nanolayers act as planar anchoring surfaces to the cellulose nanorods and, after evaporation of the water coloured films are obtained. The precursor solutions and the photonic films were investigated by Describe several techniques.
\end{abstract}

\section{Introduction}

Nature is an inexhaustible source of inspiration for materials science due to evolution and natural selection. Life on earth has developed extraordinary complex multifunctional molecules and materials with unique properties. Natural creatures have established distinctive functions such as directional water collection on wetted spider silk, antifogging properties of mosquito compound eyes, water capture and wing-locking device of the beetle, multicolour surface of butterfly wings, and super hydrophobicity and low adhesion of lotus leafs, among other examples [1]. Structural colours have attracted much attention in a wide variety of research fields due to the complex interactions between light and sophisticated microstructures created [2]. This mechanism forms the basis of the most stunning displays of colour in nature that can be observed

a e-mail: ana.c.trindade@ntnu.no 
in butterflies, moths, beetles, birds, fishes, plants and fruits. A beautiful example of this type of colouration can be found on the Morpho butterfly wings, which occurs due to the presence of lamellar structures in its ridges that offer a strong reflection in a given wavelength [3].

The depletion of petroleum-based resources and the increasing environmental concerns have stimulated considerable interest in the development of environmentally sustainable materials. Bio-based materials that have various advantages (e.g., renewability, biodegradability, and environmental friendliness), can be used as a good alternative. Cellulose, the most abundant renewable organic compound on earth, is a fascinating and almost inexhaustible, sustainable natural polymer that has been vastly used in our day-to-day products and applications and is now considered an alternative to fossil fuel-based polymers $[4,5]$.

Cellulose nanocrystal particles are produced through a controlled chemical treatment, usually via a strong acidic hydrolysis, where the amorphous domains are dissolved and the local crystalline contacts between nanofibrils are broken. After that, the dispersion is submitted to a high-power mechanical or ultrasonic treatment and the free acid is removed by several neutralization steps with distilled water [6]. The extraction of CNCs using this acid hydrolysis method was first described in 1949 by Rånby and Ribi [7] and results in a suspension consisting of nanorods [8]. Above a critical concentration and under suitable conditions, CNCs have a self-assembling tendency in suspension towards a chiral nematic liquid crystalline orientation [9]. The geometric dimensions of CNCs, such as length and width, may vary depending on the origin of cellulose microfibrils and the acidic hydrolysis conditions such as time, temperature, purity or cellulosic source [8].

Solid films prepared from cellulose nanocrystals present remarkable optical properties: iridescence, selective reflection of left circularly polarized light, and transmission of right circularly polarized light [10]. Fernandes et al. [11] reported sheared iridescent cellulosic films, with tuneable mechanical and structural colour properties, which mimic the structures found in plants.

Clays are fascinating materials present almost everywhere on Earth and as CNCs, clays can generate a liquid crystalline phase in water. Recently clays have become part of nanomaterials science [12,13]. Clay minerals are phyllosilicates consisting of charged nanolayers, which are made up of sheet structures based on the $\mathrm{Si}_{4} \mathrm{O}_{10}$ unit cell [12]. Sodium-Fluorohectorite ( $\mathrm{NaFh}$ ), with nominal composition $\mathrm{Na}_{\mathrm{x}} \mathrm{Mg}_{(3-\mathrm{x})}$ $\mathrm{Li}_{\mathrm{x}} \mathrm{Si}_{4} \mathrm{O}_{10} \mathrm{~F}_{2}$ ( $\mathrm{x}$ is the magnitude of the layer charge) is a smectite clay that belongs to the $2: 1$ clay family $[14,15]$. In the case of Sodium-Fluorohectorite, the chargecompensating counterion present in the interlayer space is $\mathrm{Na}^{+}[13,14]$. The nanolayers ("cards") in the dry state form nano-layered particles, so-called "decks of cards" [12-15]. NaFh belongs to the family of water swelling clays that can incorporate water at variable amount in the interlayer space [15]. NaFh can be synthesized [16] with charge homogeneity superior to their natural counterparts [16], allowing for quantitative swelling and delamination into individual nanolayers [17]. For the samples used in the present study, $\mathrm{x}=0.5$, i.e., we have been working with clays samples with nominal composition $\mathrm{Na}_{0.5} \mathrm{Mg}_{2.5} \mathrm{Li}_{0.5} \mathrm{Si}_{4} \mathrm{O}_{10} \mathrm{~F}_{2}$.

Synthetic NaFh belongs to a handful of layered compounds that show the phenomenon of osmotic swelling $[17,18]$. Contrary to liquid-phase exfoliation, e.g., with graphene that involves brute force sonication, osmotic swelling is a thermodynamically allowed process [19]. It allows for a gentle delamination, preserving the aspect ratio inherent in the particle diameter of the starting material. For $\mathrm{NaFh}$, nano-layers with a thickness of $1 \mathrm{~nm}$ and a median diameter of $20 \mu \mathrm{m}$ can be obtained simply by immersing the clay as synthesized material into deionized water. Due to the large aspect ratio, even very dilute suspensions $(<1 \% \mathrm{vol})$ are not isotropic but generally 
nematic [17]. Due to this large aspect ratio, even non-exfoliated NaFh particles can form nematic phases [20-22].

Previous work on CNC-clay composite materials include the work of Liu et al. who combined montmorillonite clay and nanofibrillated cellulose to prepare a clay nano-paper hybrid material for fire retardancy and gas barrier functions [23]. They added montmorillonite in order to improve the barrier properties of pure cellulose nano-paper. The data suggest that clay nano-paper is of great interest for application in self-extinguishing composites and for further development into barrier layers in packaging applications.

Initially, in this work we describe the production of cellulose nanocrystals from a commercial source, followed by the characterization (chemical, structural and dimensional) of the starting materials (i.e., the starting cellulosic material (CMC) is compared to the prepared CNC material). Then we present the analysis of solutions and thin solid films are then characterized by a range of techniques, including spectroscopy, microscopy, as well as X-ray and neutron scattering. Moreover, we present for the first time the results of addition of $\mathrm{NaFh}$ nanoclay to nanocrystalline cellulose.

\section{Materials and methods}

\subsection{Production of $\mathrm{CNCs}$ and $\mathrm{CNC} / \mathrm{NaFh}$ mixtures}

The CNC particles were prepared based on the method documented by Gray et al. [24], with minor adjustments by Fernandes et al. [10]. The cellulosic source used in this work is a commercial microcrystalline cellulose derived from cotton (MCC; Avicel@, PH-101, $50 \mu \mathrm{m}$ particle size, lot \#BCBJ0229V, Sigma-Aldrich). The microcrystalline cellulose was hydrolysed in sulfuric acid (95-97\% purity, Sigma-Aldrich), with an acid/solid ratio of 8.5:1, at $45^{\circ} \mathrm{C}$ for $40 \mathrm{~min}$ under continuous magnetic agitation. In order to cease the reaction, ultrapure water (Millipore Elix Advantage 3 water purification system) was added, with a volume of 10 times the initial volume of the reaction. The resulting material was washed, diluted and centrifuged with ultrapure water (12.000 rpm for $20 \mathrm{~min}$, Thermo Scientific Heraeus Multifuge X1R Centrifuge) until the $\mathrm{pH}$ of the CNC suspension was between 1.9 and 3.9, and then the supernatant was collected. The suspension was dialyzed in ultrapure water (using Spectrum Spectra/Por@ 4 dialysis membranes), and the water was changed every day until constant $\mathrm{pH}$ readout was obtained. The suspension was produced by submitting it to three consecutive cycles of $20 \mathrm{~min}$ of sonication (Hielscher UP400S ultrasonic homogenizer, $460 \mathrm{~W}, 24 \mathrm{kHz}$ ) over an ice bath at 0.85 of the cycle and $80 \%$ amplitude. The concentration of the aqueous CNC, $2.47 \%$ (w/w), was obtained by gravimetry. A suspension of $\mathrm{NaFh}$ at $1 \%(\mathrm{w} / \mathrm{w})$ concentration in water and the aqueous CNC suspension at $2.47 \%(\mathrm{w} / \mathrm{w})$ concentration were mixed to a final solution with $98 \%$ CNCs and $2 \% \mathrm{NaFh}(\mathrm{w} / \mathrm{w})$.

\subsection{Characterization}

Several characterization techniques were used to characterize chemically, optically, structurally and dimensionally the CNCs prepared in this work. Fourier Transform Infrared (FTIR) spectroscopy was used to chemically characterize microcrystalline and nanocrystalline cellulose used throughout this study. For this characterization a Spectrum Two FT-IR spectrometer was used, equipped with an attenuated total reflectance (Perkin Elmer), with a spectral gap from 4000 to $400 \mathrm{~cm}^{-1}$ and a resolution of $4 \mathrm{~cm}^{-1}$. 
Elemental analysis (EA) was conducted by a Flash EA 1112 CHNS series elemental analyser from Thermo Finnigan-CE Instruments. Using a NETZSCH STA 449 F3 Jupiter® thermal analyser, differential scanning calorimetry with thermogravimetric analysis (DSC-TGA) was performed. To determine the caloric effects and mass change, the samples, $2 \mathrm{mg} \mathrm{w} / \mathrm{w}$, were heated, open crucibles, at $10^{\circ} \mathrm{C} / \mathrm{min}$ from 25 to $550{ }^{\circ} \mathrm{C}$ in an inert nitrogen atmosphere.

In order to map the surface of solid CNC films derived from droplets, we used the profilometry characterization technique. This measurement was made with an AlphaStep® D-600 Stylus Profiler from KLA Tencor. The specifications of the scanning speed were $0.2 \mathrm{~mm} / \mathrm{s}$, while the stylus force applied was $1 \mathrm{mg}$.

The morphology was examined by scanning electron microscopy (SEM), with a Carl Zeiss Auriga CrossBeam (SEM-FIB) system, equipped with an Oxford energy dispersive X-ray spectrometer. The images were acquired with an acceleration voltage of $5 \mathrm{kV}$ and an aperture size of $30 \mu \mathrm{m}$. The characterized samples were glued with a double-sided tape to a sample holder and were subsequently coated with a mixture of gold and carbon using a Q300T D sputter coater from Quorum Technologies.

Atomic Force Microscopy (AFM) allowed to determine the mean dimensions of length and width of the CNCs. The equipment used was an Asylum Research MFP$3 \mathrm{D}$ in tapping mode with commercial silicon probes (scanning frequency of $300 \mathrm{kHz}$, $k=26 \mathrm{~N} / \mathrm{m}$ ). The samples to be characterized were submitted to a preparation process consisting in the sonication (using a Hielscher UP400S, $460 \mathrm{~W}, 24 \mathrm{KHZ}$, at 0.85 of the cycle and $80 \%$ amplitude) of a diluted aqueous CNC suspension $(0.01 \% \mathrm{w} / \mathrm{w})$ on an ice bath during two consecutive runs of $10 \mathrm{~min}$. Immediately after the sonication process, $1 \mu \mathrm{l}$ droplets of the diluted suspension were deposited onto a mica substrate (Muscovite Mica V-5 from Electron Microscopy Sciences). The acquired AFM images were analysed via the software Gwyddion (version 2.53, http://gwyddion. net), which consisted in 150 manual measurements of length and width of CNC particles.

X-Ray Diffraction (XRD) spectra were measured using an X-Ray diffractometer (PANalytical XPert PRO MRD) with Bragg-Brentano $(\theta / 2 \theta$ coupled) with CuK- $\alpha$ $(1.54 \AA)$ radiation. The scanning step during the characterization was $2 \theta=0.003$, from $10^{\circ}$ to $40^{\circ}$.

The width of the prepared films was measured with a Mitutoyo Digimatic Micrometer Series 293. Each film was measured at least 10 times in different positions. Macroscopic photographs of films (from both droplets and suspensions) were taken with a Canon 550D camera, outfitted with a Canon EF-S $60 \mathrm{~mm}$ macro lens under visible light. Left and right circular polarizers and cross polarizers were also used to characterize the reaction of the samples under visible light. Polarized Optical Microscopy (POM) images were recorded and analysed using an Olympus BX-51 microscope connected to a cold light source (Olympus KL2500) with an attached camera (Olympus DP73) together with Olympus Stream Basic 1.9 software. Using the program Lightscan 1.1.17 in combination with the microscope, the spectra of reflectance of visible light of the samples were obtained. The solid iridescent films were observed under linear and circularly polarized light.

The SANS-instrument at the JEEP-II reactor at IFE was employed for the smallangle neutron scattering (SANS) measurements. Samples for SANS were prepared by dissolving nanocellulose powder in heavy water $\left(\mathrm{D}_{2} \mathrm{O}\right)$ with subsequent stirring $(1 \mathrm{hr})$ and ultrasonication $(20 \mathrm{~min}) . \mathrm{D}_{2} \mathrm{O}$ was used instead of $\mathrm{H}_{2} \mathrm{O}$ order to obtain a low background and good contrast in the SANS measurements. Sample concentrations of $5 \%(\mathrm{w} / \mathrm{w})$ and $1 \%(\mathrm{w} / \mathrm{w})$ were employed, and in addition measurements were done with the $5 \%(\mathrm{w} / \mathrm{w})$ sample subjected to a horizontal magnetic field $(1 \mathrm{~T})$. Two different sample-detector distances $(1.0 \mathrm{~m}$ and $3.4 \mathrm{~m})$ and two different neutron wavelengths ( $5 \AA$ and $10 \AA$ ) were used in order to obtain a large total 

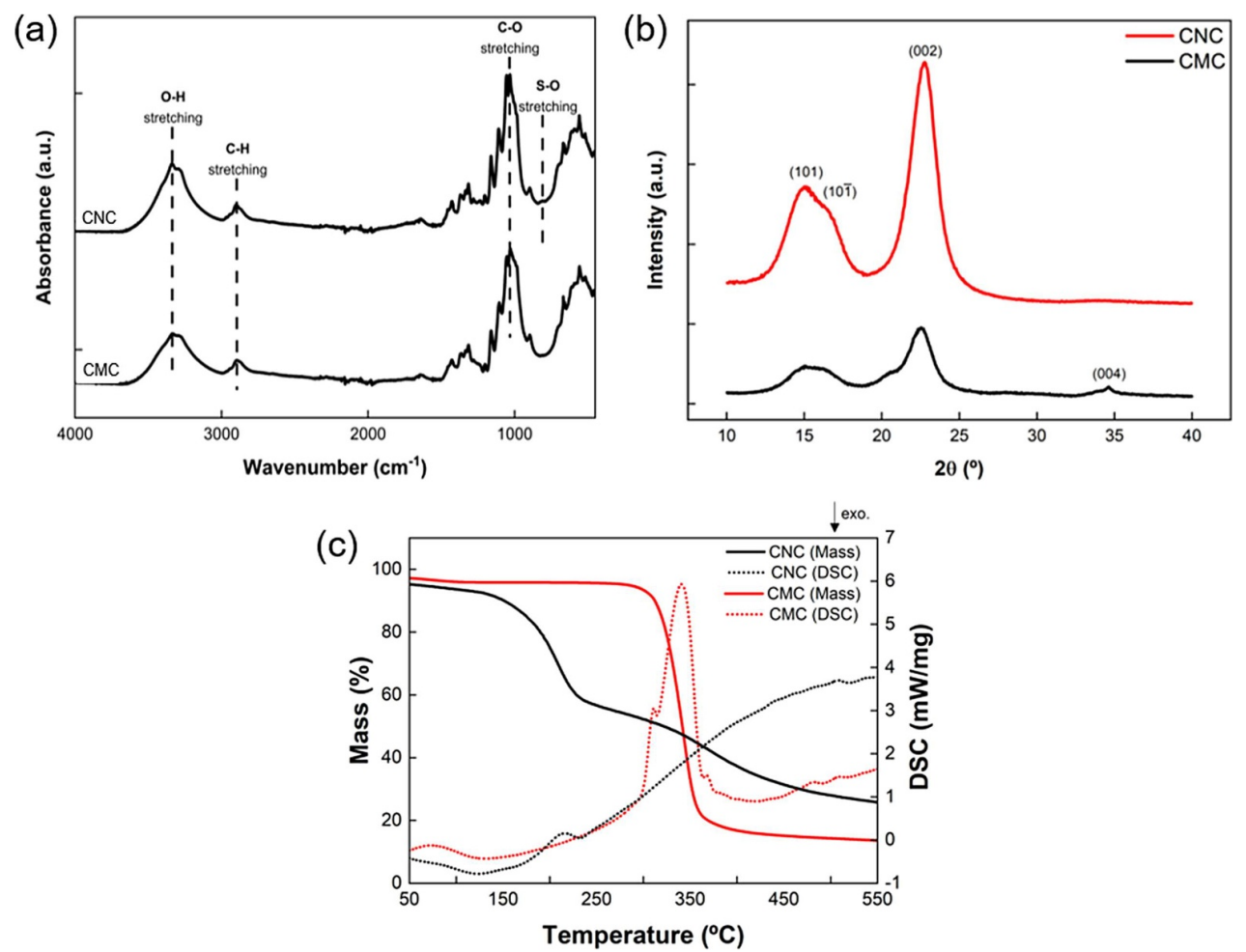

Fig. 1. (a) FTIR spectra of CMC and CNC samples that were analysed. (b) XRD diffractograms of the produced CNCs (red line) and the starting material CMC (black line). (c) DSC-TGA analysis of cellulose nanocrystals (black lines) and microcrystalline cellulose (red lines).

scattering range (q-range). The transmission was measured separately, and the scattering was normalized to absolute units $\left(\mathrm{cm}^{-1}\right)$ by taking into account empty cell and background scattering. All samples were inspected before being introduced into $2 \mathrm{~mm}$ quartz cuvettes. The temperature used during the measurements was $23^{\circ} \mathrm{C}$.

\section{Results and discussion}

\subsection{Chemical, structural and morphological characterization of CNCs}

The produced cellulose nanocrystals and the starting cellulosic material (CMC) were characterized by means of different structural, dimensional, and chemical techniques: DSC-TGA, FTIR, AFM, XRD and EA (Figs. 1, 2 and Tab. 1). A comparison of $\mathrm{CMC}$ and CNCs is necessary to assess if the performed acidic hydrolysis reaction was successful.

The FTIR spectra of both samples (CMC and CNCs) are represented in Figure 1a, where the absorbance bands of the stretching vibrations are visible. Each individual vertical line represents stretching vibrations of the cellulose molecules. The examination of this figure allows to establish that both samples display the same cellulose characteristic bands at 3330, 2900 and $1030 \mathrm{~cm}^{-1}$, approximately, which is in accordance with the theoretical values found in literature. These bands correspond to the stretching vibrations of $\mathrm{O}-\mathrm{H}, \mathrm{C}-\mathrm{H}$ and $\mathrm{C}-\mathrm{O}$ bonds, respectively [26]. The 


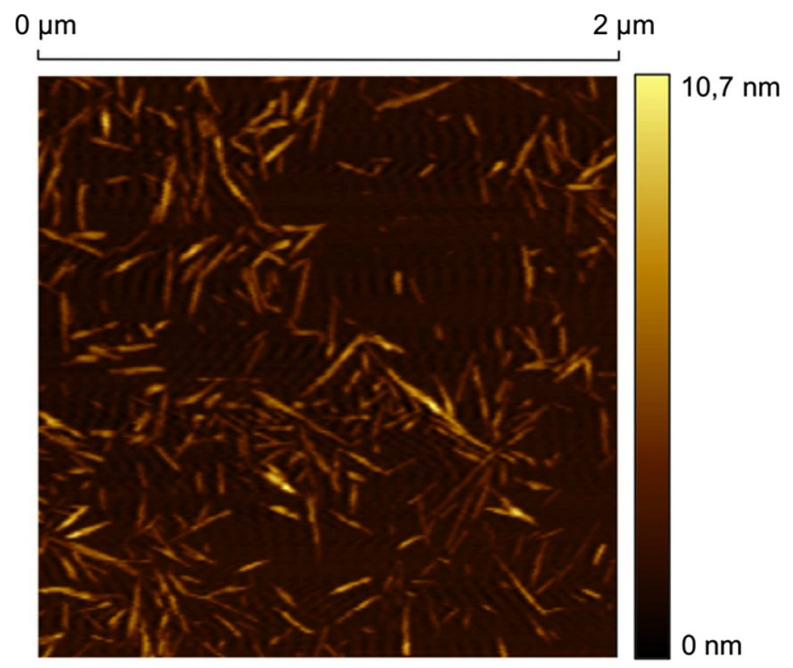

Fig. 2. AFM image from amplitude retrace mode of a diluted aqueous CNCs suspension after evaporation. Window dimensions: $2 \mu \mathrm{m} \times 2 \mu \mathrm{m}$.

Table 1. Percentage of each element (C, H, S and O) in the CMC and CNC samples as obtained by elemental analysis. The oxygen percentage was obtained by mass difference between all elements.

\begin{tabular}{lccccc}
\hline Sample & $\begin{array}{c}\text { Carbon } \\
(\%(\mathrm{w} / \mathrm{w}))\end{array}$ & $\begin{array}{c}\text { Hydrogen } \\
(\%(\mathrm{w} / \mathrm{w}))\end{array}$ & $\begin{array}{c}\text { Sulphur } \\
(\%(\mathrm{w} / \mathrm{w}))\end{array}$ & $\begin{array}{c}\text { Oxygen } \\
(\%(\mathrm{w} / \mathrm{w}))\end{array}$ & $-\mathrm{OSO}_{3} \mathrm{H} / 100$ \\
\hline $\begin{array}{l}\text { Pure Cellulose } \\
\text { predicted }\end{array}$ & 44.44 & 6.18 & - & 49.38 & - \\
values [25] & & & & & \\
\hline CMC & & & & & - \\
\hline CNCs & 42.85 & 6.30 & - & 50.85 & - \\
\hline
\end{tabular}

absorbance band at $890 \mathrm{~cm}^{-1}$ is associated with the stretching vibration of $\mathrm{C}-\mathrm{O}-\mathrm{C}$ of the $\beta(1,4)$ glycosidic linkage [27]. Another band, at $820 \mathrm{~cm}^{-1}$, corresponds to the $\mathrm{S}-\mathrm{O}$ bond stretching [28]. This last band can only be observed in the spectra of the CNC sample and was only visible in samples that went through an acidic hydrolysis process (in other words, that have sulfate groups present in the cellulose structure).

The X-ray diffractograms of the synthetized CNCs and the starting CMC material are displayed in Figure 1b. The data show a main peak at $2 \theta=22.7^{\circ}$, which is consistent with crystalline the 002 plane. Two smaller peaks can be observed at $2 \theta=15.0^{\circ}$ and $2 \theta=16.6^{\circ}$ corresponding to the 101 and planes, respectively [29]. These results correspond to the characteristic peaks of cellulose type I, which allow to conclude that the cellulose structure remains stable after the acidic hydrolysis process [30]. In the diffractogram pattern of the CMC sample there is also a small peak at $2 \theta=34.6^{\circ}$, which corresponds to the 004 plane.

Using the data from the diffractogram patterns, the crystallinity index ( $\mathrm{CrI}$ ) of both CMC and CNC samples can be determined employing the documented empirical method of Segal et al. [31], where the CrI can be calculated using the extracted data from Figure 1a in combination with equation (1):

$$
\mathrm{CrI}=\frac{\left(I_{(002)}-I_{\mathrm{am}}\right)}{I_{(002)}} \times 100 .
$$


In this equation, $I_{(002)}$ corresponds to the maximum diffraction intensity from the 002 peak at a $2 \theta$-angle between $21^{\circ}$ and $23^{\circ} . I_{\text {am }}$ corresponds to the minimum diffraction intensity of the amorphous region at a $2 \theta$-angle between $18^{\circ}$ and $20^{\circ}$. The crystallinity index obtained for the samples was $75.2 \%$ for CMC and $89.8 \%$ for CNCs. As expected, the CrI of the CNCs increased in comparison to that of the CMC sample due to the acidic hydrolysis process in which the amorphous regions were removed. These results are in accordance with the values found in the literature for a cotton source [28]. Hoeger et al. [32] presented an identical crystalline index for CNCs from Avicel PH-101 (88\%) using similar hydrolysis conditions (65\% sulfuric acid, $\left.55^{\circ} \mathrm{C}, 30 \mathrm{~min}\right)$.

The DSC-TGA analysis of both cellulose nanocrystals (CNCs) and the CMC microcrystalline cellulose is presented in Figure 1c, allowing analysis of the thermal stability before and after the acidic hydrolysis. On both samples, all mass changes before $100^{\circ} \mathrm{C}$ occurred as a result of the evaporation of residual water that remained in the cellulose fibres. The CMC sample shows a single sudden decline in the TGA curve between $260^{\circ} \mathrm{C}$ and $360^{\circ} \mathrm{C}$ as a result of mass loss of cellulosic material and a series of degradation processes that take place, such as dehydration, decomposition and depolymerization of the glycoside units [33,34]. These results are confirmed by DSC measurements. The CNCs sample had been subjected to an acidic hydrolysis process, performed with $\mathrm{H}_{2} \mathrm{SO}_{4}$, which means that the degradation process becomes a second order pyrolysis reaction with two mass change events. A sharp mass drop, the first degradation step in the CNCs sample, can be observed at temperatures between $130^{\circ} \mathrm{C}$ and $240{ }^{\circ} \mathrm{C}$. The direct after-effect to this step is the degradation of the regions that are more accessible to sulphate groups $[33,35]$. The second degradation step corresponds to the degradation breakdown of the more crystalline regions of the sample, which are less susceptible to the hydrolysis process [33]. Beyond $400{ }^{\circ} \mathrm{C}$ cellulose oxidation occurs, leading to the formation of carbon-based residues of the samples [34].

The dimensional characterization of the CNCs sample was done by AFM imaging techniques, cf. Figure 2. The acquired AFM image shows a dehydrated droplet of the diluted aqueous CNC suspension $(0.01 \%(\mathrm{w} / \mathrm{w}))$. In order to assess the lateral dimensions of the "rice-like" CNC structures, 150 measurements were made using the Gwyddion software. The mean values of the lengths and widths measured were $182 \pm 34 \mathrm{~nm}$ and $5 \pm 1 \mathrm{~nm}$, respectively, which correspond to an aspect ratio of 36 . The obtained length is within the range presented by Fernandes et al. (152 $\pm 65 \mathrm{~nm})$ [10] and in accordance to the values listed by Rochas and co-workers for similar hydrolysis conditions (65\% sulfuric acid, $45^{\circ} \mathrm{C}$ and $30 \mathrm{~min}$ ) [36].

The amount of sulphate ester groups in the anhydroglucose units present in the CNC chains can be quantified by the elemental analysis (EA) technique. Table 1 shows the collected data regarding the percentage of carbon, hydrogen and sulphur of each sample in addition to the predicted values for pure cellulose and the calculated value $(\mathrm{n})$ of the average degree of substitution of the $\mathrm{OSO}_{3} \mathrm{H}$ anhydroglucose units.

From the data obtained with the EA technique, it is possible to conclude that, as expected, the CMC sample does not have sulphur present in its chain. Using these data from Table 1 in combination with the method developed by Hamad et al. [37] and given in equation (2), it is possible to determine the value (n) of the degree of substitution of the $-\mathrm{OSO}_{3} \mathrm{H}$ anhydroglucose units according to the formula:

$$
\mathrm{n}=(100 \times 162 \times S(\%)) /(3206-80 \times S(\%)) .
$$

Here $\mathrm{S}(\%)$ is the measured value of sulphur by elemental analysis. The sulphur content of the CNCs sample is $0.76 \%$, which is equal to the value presented by Hoeger et al. [32] for similar hydrolysis conditions. The value of $\mathrm{n}$ for the $-\mathrm{OSO}_{3} \mathrm{H}$ anhydroglucose units for the CNC sample is calculated to 3.92, which is slightly lower than the value 4.39 published by Fernandes et al. [10,11]. This discrepancy is probably due to the 

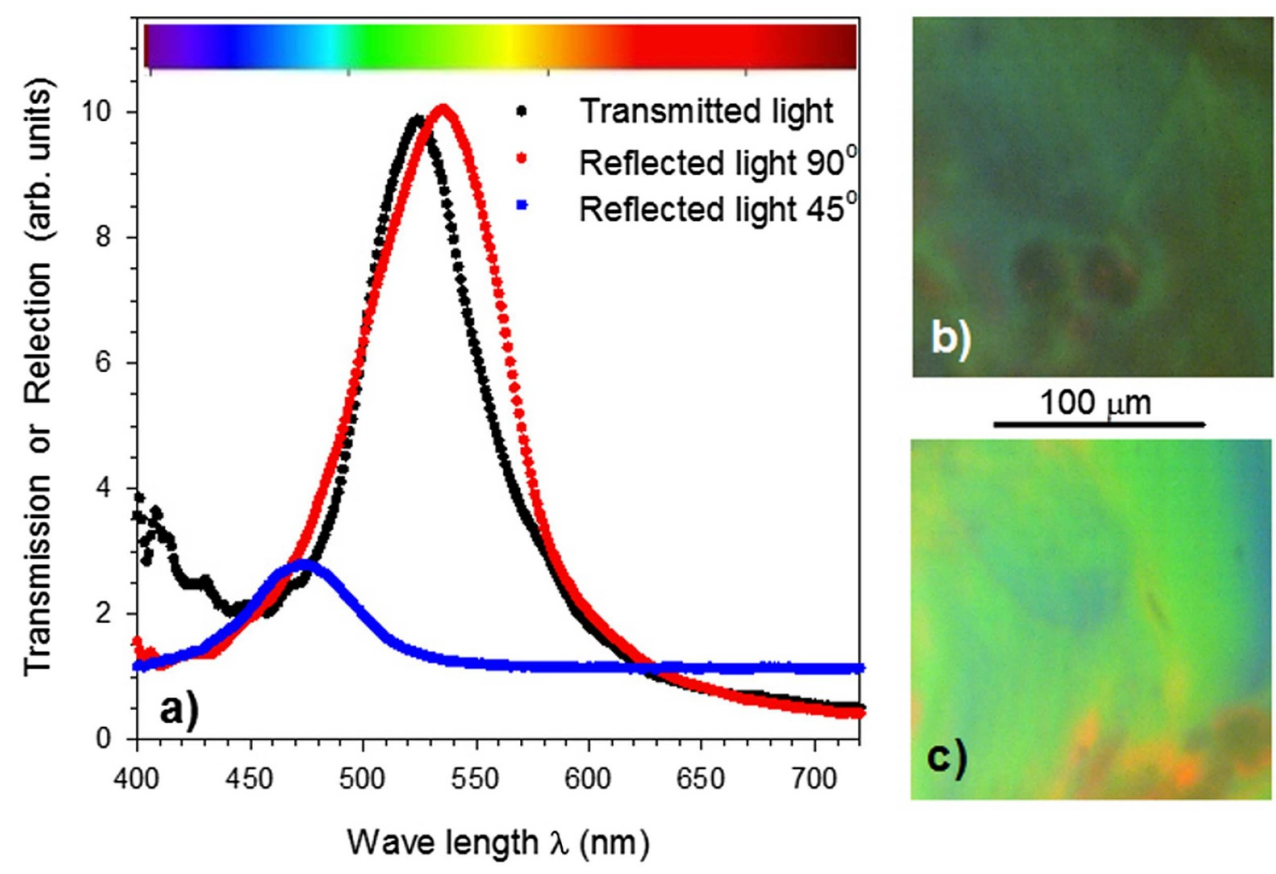

Fig. 3. (a) Transmission spectrum and reflection spectra measured at light incidence angles of $90^{\circ}$ and $45^{\circ}$. The coloured band shows an approximate rendering of the visible spectrum. (b) and (c) Optical microscope images of the same sample in $90^{\circ}$ transmitted and reflected light, respectively.

hydrolysis time (130 min) used by these authors, since longer reaction time translates into a greater degree of substitution and therefore a higher value of $n$.

Droplets with $10 \mu \mathrm{l}$ of the CNC suspensions were drop-cast on microscope slides using Easy $40+$ micropipette in order to produce thin films by solvent evaporation at room temperature. The obtained thin films of CNCs on glass plates were studied by optical microscopy. The diameters of the nearly circular areas were about $15 \mathrm{~mm}$, and the thickness was about $20 \mu \mathrm{m}$. Figure 3 shows an example of a low-magnification microscope image of a film. Near the outer edge, red, yellow and green colours can be seen, but closer to the centre the colour is strong blue with some tiny green spots in between.

In order to record the colour spectrum, samples were mounted in a microscope (Nikon Optiphot) with a spectrometer (Redtide USB650, Oceanoptics.com) attached to the C-mount port of the microscope via an optical fibre. The spectra were analysed using the OceanView software. The intensity of the light sources was adjusted to use the full intensity range of the spectrometer, and typically the acquisition time for a spectrum was set to $200 \mathrm{~ms}$. The samples were studied both in transmitted and reflected light with $90^{\circ}$ incidence angle of the light. The microscope light source used a halogen lamp (Osram XenoPhot HLX) with colour temperature $3350 \mathrm{~K}$. The spectrum of the light source was used as references for calculating the optical transmission and reflection spectra. For both types of spectra, a set of crossed linear polarizers were used to remove diffuse scattered white light.

In this configuration the films showed clear green or green-blue colours with some spots of red and orange in between. Figure 3a shows the calculated transmission and reflection spectra based on the light intensity measurements, and Figures $3 \mathrm{~b}$ and $3 \mathrm{c}$ show microscope images of the measured areas in the film. The spectrometer field of 


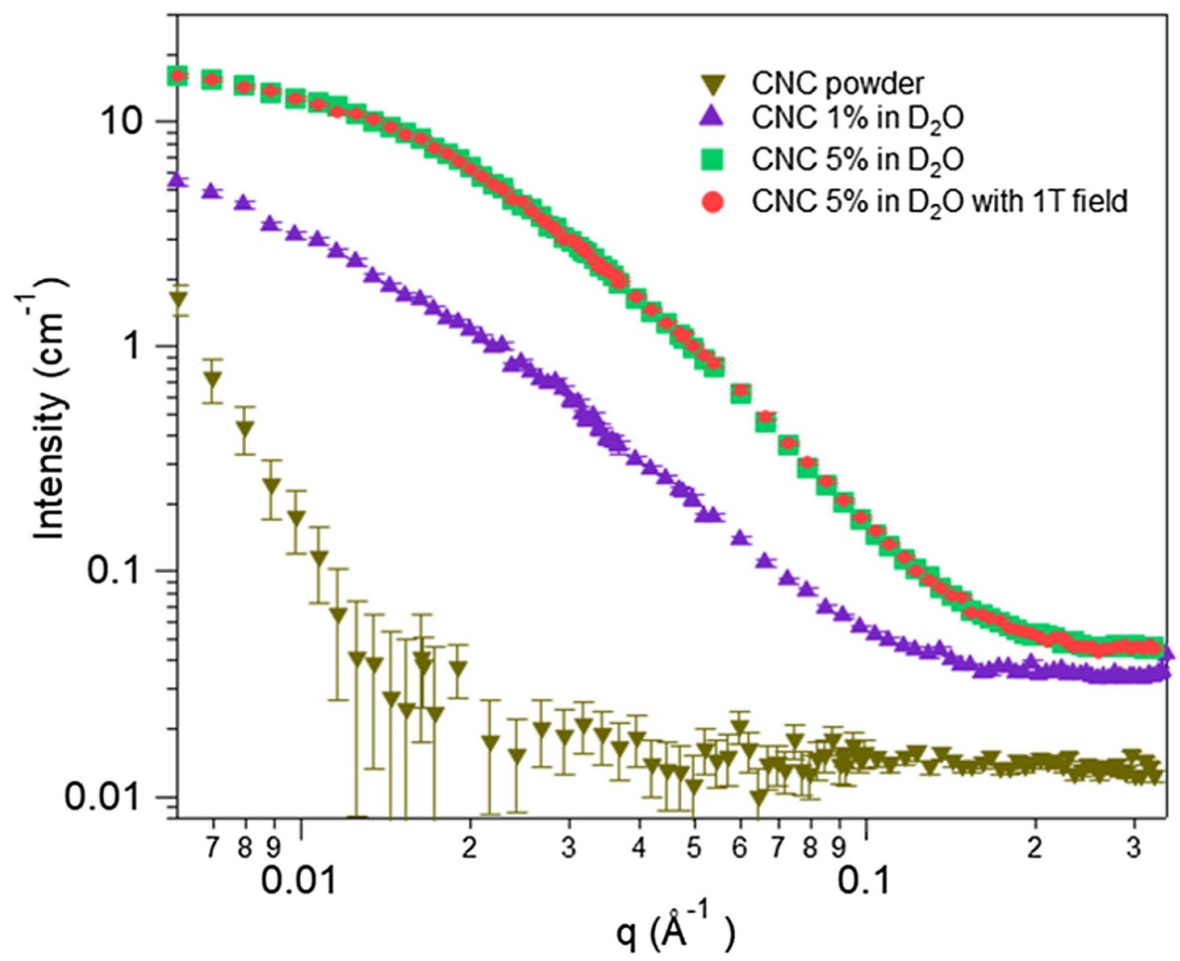

Fig. 4. SANS patterns from cellulose nanocrystals in heavy water $\left(\mathrm{D}_{2} \mathrm{O}\right)$ at varying concentrations. Also, the scattering from dry CNC powder is shown for comparison.

view is circular with a diameter of about $100 \mu \mathrm{m}$. As seen, the transmission spectrum is slightly shifted toward a shorter wavelength $\lambda$ than that for the reflection, with peak values at $\lambda=525 \mathrm{~nm}$ and $535 \mathrm{~nm}$, respectively. The same sample was also mounted on the microscope with incidence and reflection angles both equal to $\varphi=65^{\circ}, 45^{\circ}$, and $30^{\circ}$. A main broad peak in the reflection spectrum was then located in the range $415 \mathrm{~nm} \leq \lambda \leq 475 \mathrm{~nm}$ corresponding to a dark blue colour as can be seen in Figure 3a for $\varphi=45^{\circ}$. The optical images showed a mixture of $\mu \mathrm{m}$-sized domains in various shades of blue and green, which may be the reason for relatively wide peaks. Colour differences may be partly due to misalignment of the cellulose nanocrystals relative to the glass surface beneath. Using the CIE 1931 colour space [34], the $45^{\circ}$ reflected light spectrum in Figure 5a could be characterized with coordinates $(x, y)=(0.30,0.31)$ in the CIE xy-chromaticity diagram. The green colours for $90^{\circ}$ reflection, as in Figure $3 \mathrm{c}$, were closer to $(x, y)=(0.38,0.48)$.

\subsection{Characterization of CNCs and NaFh solutions by small-angle neutron scattering}

The CNCs was also characterized by means of small-angle neutron scattering (SANS). Solutions from CNCs in heavy water $\left(\mathrm{D}_{2} \mathrm{O}\right)$ with different concentrations $(1 \%$ and $5 \%$ $(\mathrm{w} / \mathrm{w}))$ were prepared, and the small-angle neutron scattering patterns are presented in Figure 4. The scattering from dry CNC powder is also shown for comparison.

We observe strong scattering from the solutions, and only weak scattering from the CNC powder. This is mainly due to the difference in contrast, since CNCs in 

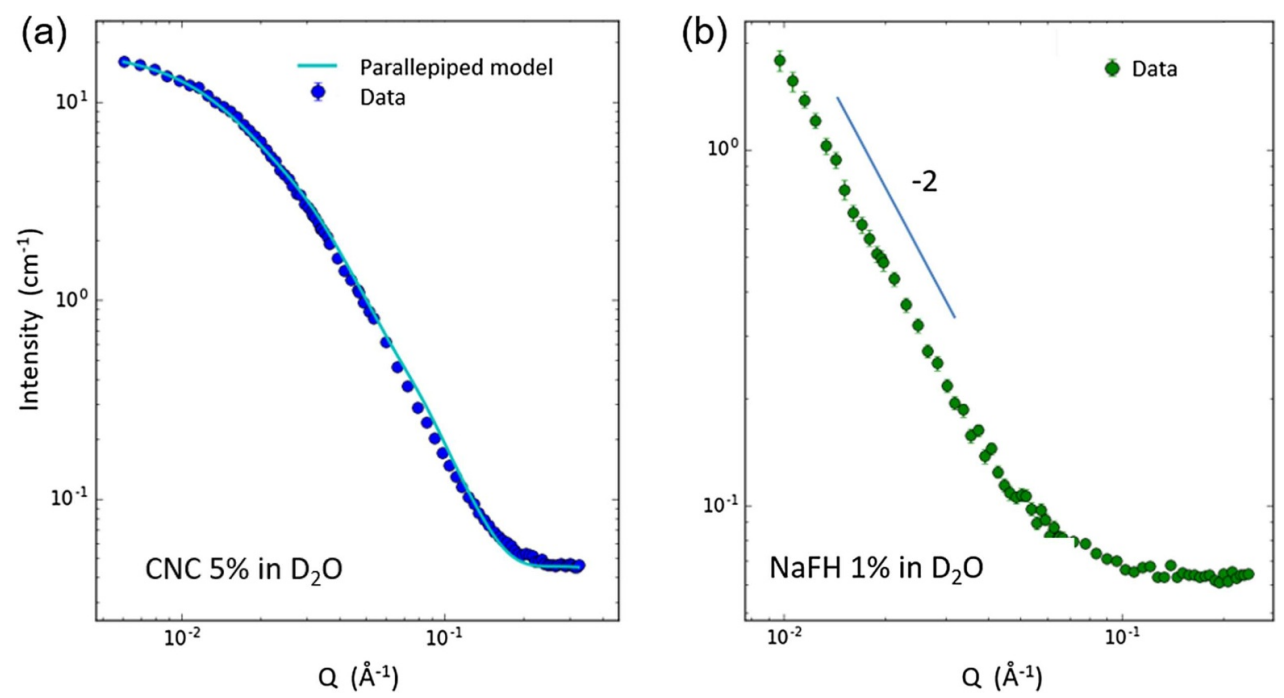

Fig. 5. (a) SANS measurement of scattered neutron intensity as function of scattering vector $\mathrm{q}$ for nanocellulose $(\mathrm{CNC})$ at $5 \%$ concentration in $\mathrm{D}_{2} \mathrm{O}$ and (b) data from a suspension of NaFh clay particles. The data from nanocellulose has been fitted to a parallelepiped particle model, whereas the clay data show the typical -2 slope (log-log scales) for extended plateletshaped particles.

water (here $\mathrm{D}_{2} \mathrm{O}$ ) gives a large difference in scattering length density between the particles and surroundings, whereas this difference is much smaller in air.

The solution patterns are typical for particles with at least one of the dimensions in the $\mathrm{nm}$ range. The SANS signal $(I)$ can generally, to a first approximation, be expressed simply as $I=C \cdot P(q) \cdot S(q)$, where $C$ is a constant proportional to the concentration, $P(q)$ is the particle form factor (representing the size and shape of the particles), and $S(q)$ is the structure factor (representing interactions between particles). If the particle interaction is negligible, the intensity in the low-q limit will be directly proportional to the concentration. As expected, the $5 \%$ sample scatters significantly stronger than the $1 \%$, but the increase is less than a factor of 5 . This is an indication of a slight interaction between the particles, since the structure factor will produce a depression of the signal at low scattering vector $q$. There are reports from literature $[38,39]$ that nanocellulose particles can be partly aligned under a magnetic field, due to diamagnetic interaction. SANS is highly sensitive to any ordering in the sample, since this can be detected as an angular variation in the intensity over the 2 D-detector. For this reason, we also collected SANS data on the 5\% sample when subjected to a field of 1 Tesla. As can be seen from the figure, there is full overlap for the data measured without and with magnetic field. Thus, no restructuring due to the field can be observed on the length scale accessible by SANS (below ca $100 \mathrm{~nm}$ ) for the moderate field strength used in this experiment. This does not exclude that magnetic ordering can take place also for this system - e.g. by employing a considerable higher field strength and/or concentration - in line with the existing variability in threshold values, as commented in reference [36].

The scattering profile could be fitted quite well to a parallelepiped model (see Fig. 5a), i.e., a particle with rectangular cross-section and a length significantly larger than the two other dimensions. This is in line with the observations of Mao et al. [25] for the nanocellulose investigated in their study. The dimensions extracted from the model were 3 and $12 \mathrm{~nm}$ for the cross-sectional size, and these dimensions are quite 
similar to those discussed by Mao et al. for one of their nanocellulose types, i.e. 3 and $20 \mathrm{~nm}$. Note that in this analysis, also a structure factor $S(q)$ was included in the modelling to take into account the interaction between particles, as commented on above. For this purpose, a so-called Hayter-Penfold structure factor [40] was used, in order to include electrostatic repulsion due to the negative charge on the surface of the CNC particles.

From SANS one can also get a rough estimate of the lower limit of the particle length. This value was estimated to ca. $90 \mathrm{~nm}$ based on model fit to the pattern in Figure 5a, which is considerably shorter than the length found from AFM, mentioned previously (average of $182 \mathrm{~nm}$ ). Here it should be taken into account that the low-q limit of the SANS data (ca. $0.006 \AA^{-1}$ ) is not sufficient to properly probe dimensions above $100 \mathrm{~nm}$. The fitted value for the length based on SANS data is therefore just a lower bond for these particles, and will furthermore be affected by any sample polydispersity. In conclusion, this means that AFM is much more accurate for the largest sizes, but does not have the necessary resolution for probing the cross sectional dimensions, where SANS can give detailed information. This investigation of the individual CNC particles in solution thus demonstrates that they are indeed nanosized, with two of the dimensions in the range of a few nanometres (cross-sectional sizes 3 and $12 \mathrm{~nm}$ ), and the third dimension well above 100 nanometres (average length $182 \mathrm{~nm}$ ). These are the primary building elements for the macroscopic samples that were studied with other techniques, e.g., optical microscopy as described above.

In Figure 5b is shown the SANS pattern from a suspension of Na-fluorohectorite clay. These particles have a large aspect ratio (lateral size towards micrometers), and in suspension they behave as independent platelet-shaped entities (disks) when the concentration is not too high (around $1 \%(\mathrm{w} / \mathrm{w})$ or lower). In contrast to the nanocellulose suspension, we do not observe any plateau-like behavior at low q-values for the clay suspension, demonstrating that the size (diameter) of the clay particles is on average larger than the size limit of the current SANS-setup (around $100 \mathrm{~nm}$ ). Furthermore, the log-log slope of the pattern is found to be close to -2.0 , which is the expected value for disk-like particles.

The SANS patterns of nanocellulose and clay are shown in comparison here to highlight the clear differences in scattering patterns from individual particles of these two systems. In a situation where nanocellulose is mixed with clay and the solvent left to evaporate, there will obviously be strong interactions between the two particle types, as well as between the particles and the supporting medium, and other techniques than SANS must be used to inspect the resulting structures.

\subsection{Characterization of solid coloured films}

In order to analyse the effect of the addition of $\mathrm{NaFh}$ particles on the properties of the films based on CNCs, solid films were also prepared from CNCs $+\mathrm{NaFh}$ solutions. The IR- and X-ray spectra shown in Figures $6 \mathrm{a}$ and $6 \mathrm{~b}$ indicate that the characteristic bands of CNCs are still present and the crystallinity of the CNC nanorods are not much affected by the presence of the clay nanoparticles. However, the retardant effect of the clay nanoparticles in the mixture was detected as a shift of the DSC-TGA peaks toward higher temperatures, as seen in Figures $6 \mathrm{c}$ and $6 \mathrm{~d}$, which is an indication that a small amount of $\mathrm{NaFh}(2 \% \mathrm{w} / \mathrm{w})$ is enough to promote a fire-retardant behaviour of the composite. The presence of the clay nanoparticles also affects the roughness of the films at the micro/nano scale. AFM images reveal a smooth surface (Figure 7) that was also observed in SEM images as seen in Figures $8 \mathrm{c}$ and $8 \mathrm{~d}$. Figure 7a shows an AFM scan of a $3 \times 3(\mu \mathrm{m})^{2}$ flat region of a typical film. Two height profiles from this image are shown in Figure $7 \mathrm{~b}$. There are local height variations of the order of $10 \mathrm{~nm}$ over typical horizontal scales of $100-200 \mathrm{~nm}$. 

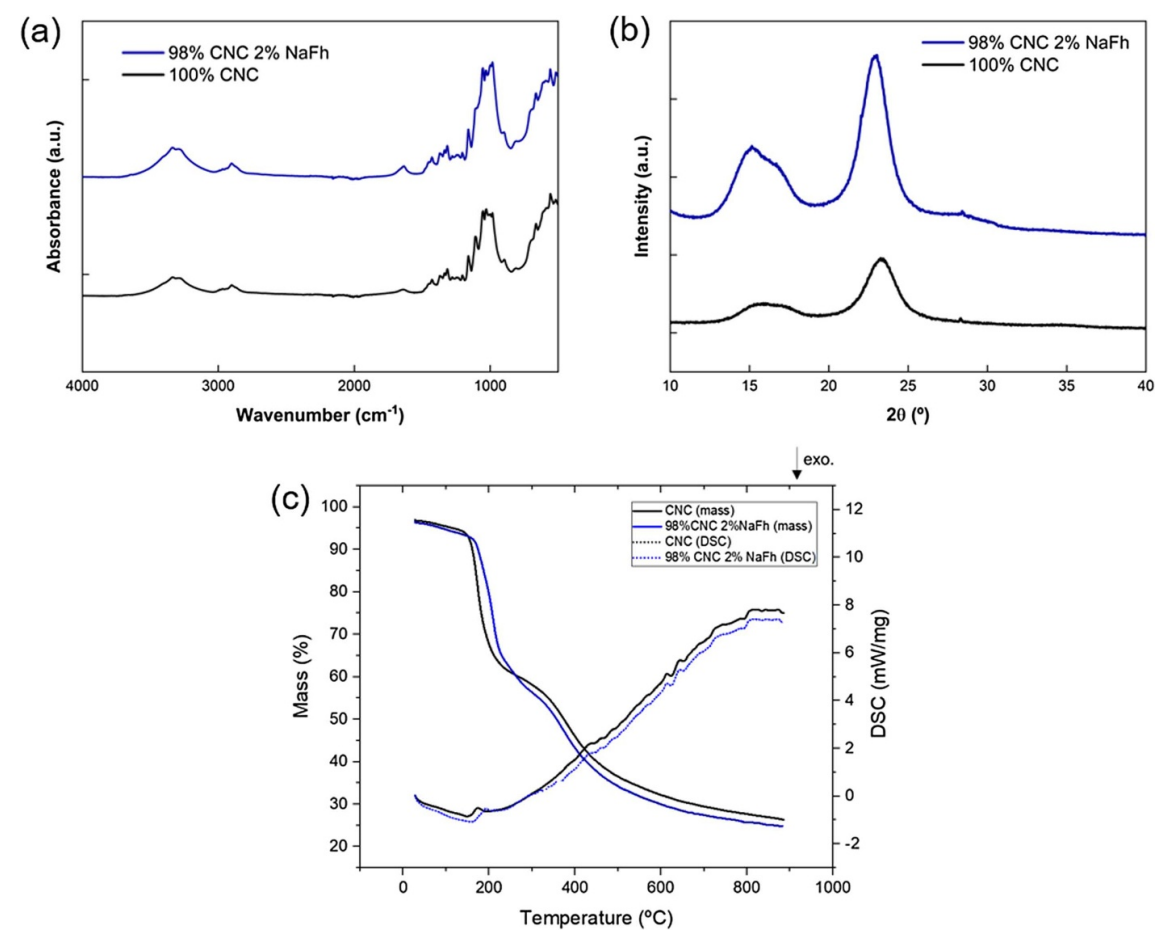

Fig. 6. CNCs and CNC/NaFh samples characterized by (a) FTIR spectra, (b) XRD diffractograms, (c) TGA analysis and DSC analysis.

(a)

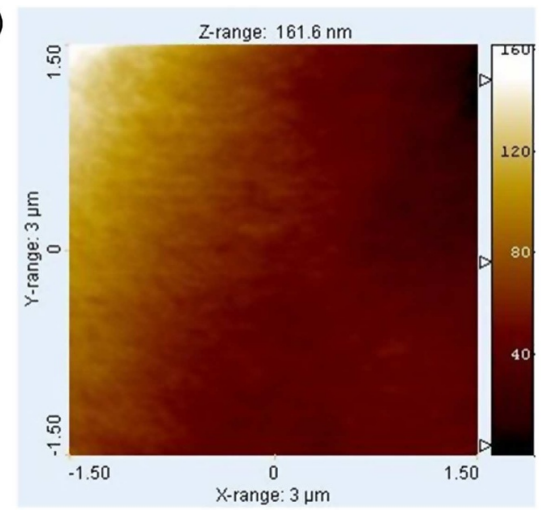

(b)

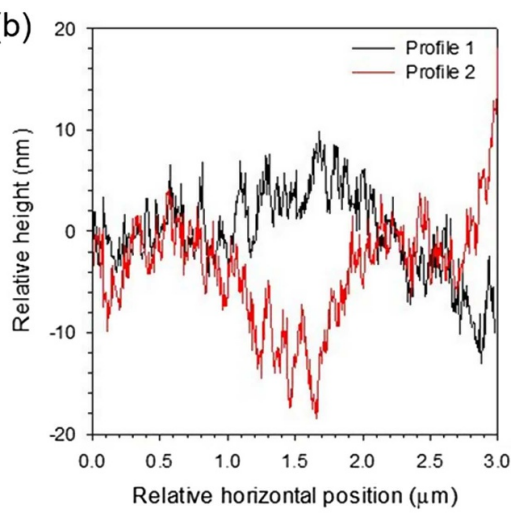

Fig. 7. (a) AFM scan of a $3 \times 3(\mu \mathrm{m})^{2}$ area of a CNC-clay film. The colour coded heights are in $\mathrm{nm}$. (b) Two height profiles from this image.

One of the most important results is related to the structural colouration of the films, which is still present for the amounts of nano-clays used in the present experiments. A "coffee-ring" effect was observed for both samples, with and without nano-clays as shown in Figures $8 \mathrm{~b}$ and 8a, respectively. The presence of the $\mathrm{NaFh}$ sheets favours the appearance of a pixelated texture at the border of the sample with colours shifted toward longer wavelengths when compared to the centre of the sample. The clay nanosheets do not destroy the cholesteric arrangement of the CNCs in solid films, and do not influence the handeness of the structure, but tend to decrease the values of the cholesteric pitch. This suggests a planar anchoring of the CNCs at the 

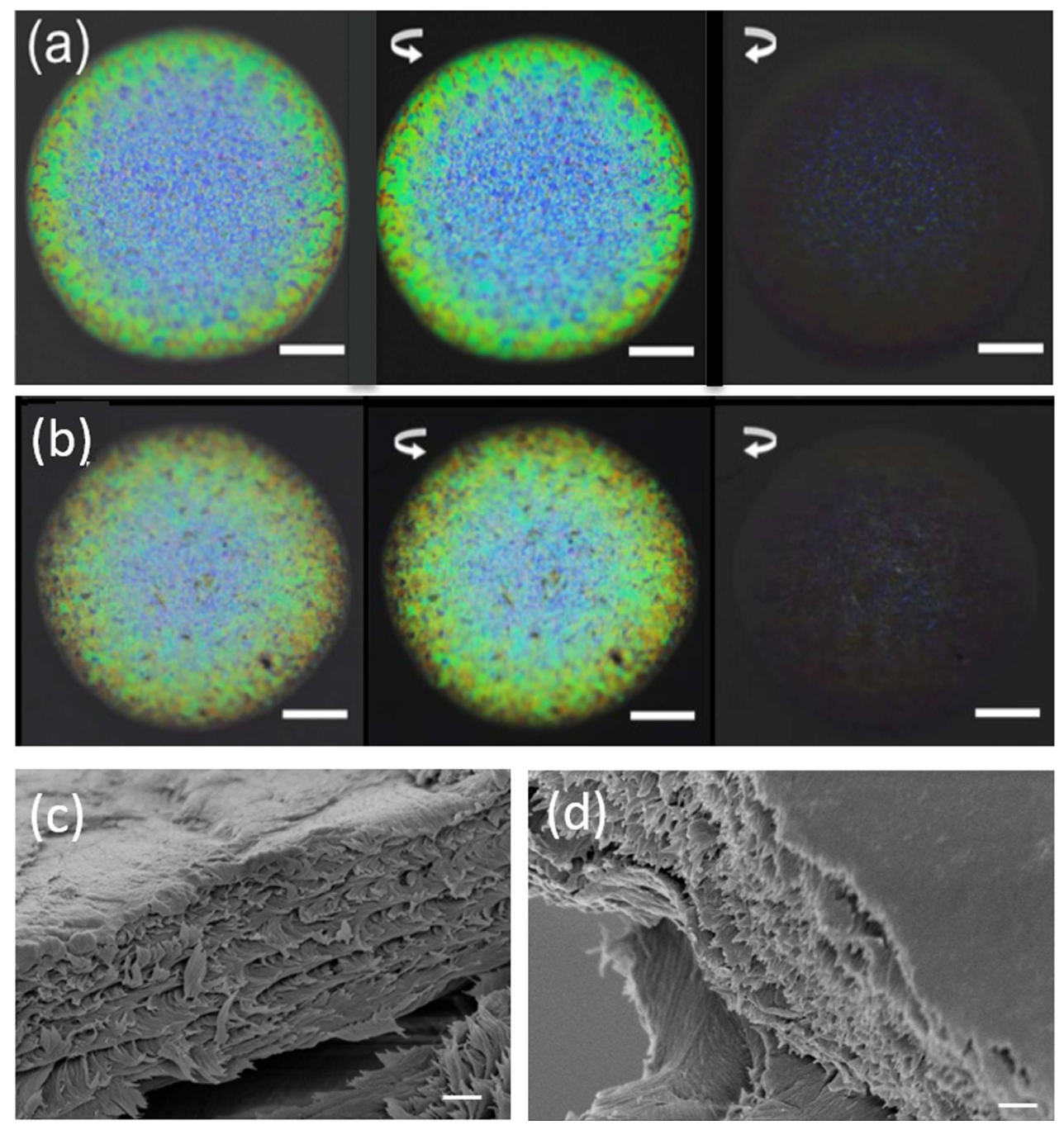

Fig. 8. A slightly tilted film seen at low magnification in an optical microscope as observed under visible light, left circularly and right circularly polarized light, from left to right, respectively, for (a) CNCs and (b) $\mathrm{CNC} / \mathrm{NaFh}$. SEM images obtained for the cross section of the films: (c) CNC and (d) CNC/NaFh. Scale bars: (a, b) $7.5 \mathrm{~mm}$; (c, d) $400 \mathrm{~nm}$.

surface of the nano-clay and a formation of a layered structure of CNCs (cholesteric layers) and $\mathrm{NaFh}$ nanosheets, corroborated by the SEM cross section image shown in Figure 8d.

\section{Conclusions}

This work shows that CNCs and $\mathrm{NaFh}$ aqueous systems are compatible. CNC water solutions were investigated, and photonic solid films were prepared. The photonic characteristics of CNC films were preserved when $2 \%$ by weight of $\mathrm{NaFh}$ was added to the water solution. The photonic solid films prepared from the mixture of CNCs and NaFh show iridescence and present left-handed nano-structures. The layered 
structuring was preserved, as shown in the cross-section images obtained by SEM. The quantity of NaFh used does not prevent the formation of a "coffee ring" effect, which translates into the development of concentric pixelated rings that change colour from the centre to the border of the sample, from blue, to green/reddish.

The material obtained will typically have fire-retardant properties, in line with what is known from other types of clay-based materials. There may therefore also be a correlation between the colour observed and specific properties, such as the degree of fire-retardation, as an example. Such properties will likely depend on the exact clay-type used, the relative concentration of CNCs and clay, as well as the overall concentration of material in the prepared suspension. The present initial work does not encompass such studies, but these possibilities are among those we plan to explore in later investigations.

Open access funding provided by NTNU Norwegian University of Science and Technology (incl St. Olavs Hospital - Trondheim University Hospital). This work was funded by FEDER funds via the COMPETE 2020 Program and National Funds through the FCT-Portuguese Foundation for Science and Technology under project numbers POCI-01-0145-FEDER007688 (Reference UID/CTM/50025), PTDC/FIS-NAN/0117/2014, PTDC/CTM-BIO/ 6178/2014, M-ERA-NET2/0007/2016 (CellColor) and PTDC/CTM-REF/30529/2017 (NanoCell2SEC). Authors also want to thank Research Council of Norway - Nano2021 Program (250619 Graphene-NanoClay Systems).

Open Access This is an open access article distributed under the terms of the Creative Commons Attribution License (http://creativecommons.org/licenses/by/4.0/), which permits unrestricted use, distribution, and reproduction in any medium, provided the original work is properly cited

Publisher's Note The EPJ Publishers remain neutral with regard to jurisdictional claims in published maps and institutional affiliations.

\section{References}

1. S. Niu, B. Li, Z. Mu, M. Yang J. Zhang, Z. Han, J. Bionic Eng. 12, 170 (2015)

2. S. Kinoshita, in Structural Colors in the Realm of Nature (World Scientific Publishing Co. Pte. Ltd., Singapore, 2008), Vol. 136

3. S. Tadepalli, J.M. Slocik, M K. Gupta, R.R. Naik, S. Singamaneni, Chem. Rev. 117, $12705(2017)$

4. J. George, S.N. Sabapathi, Nanotechnol. Sci. Appl. 8, 45 (2015)

5. J.P. Borges, J.P. Canejo, S.N. Fernandes, M.H. Godinho, in Nanocellulose Polymer Nanocomposites: Fundamentals and Applications (Wiley, 2014), p. 215

6. H. Kargarzadeh, M. Ioelovich, I. Ahmad, S. Thomas, A. Dufresne, in Handbook of Nanocellulose and Cellulose Nanocomposites (Wiley-VCH, 2017), Vol. 1, p. 1

7. S. Beck-Candanedo, M. Roman, D.G. Gray, Biomacromolecules 6, 1048 (2005)

8. M.S. Reid, M. Villalobos, E.D. Cranston, Langmuir 33, 1583 (2017)

9. U. Gill, Mechanical Properties of Cellulose Nanocrystal Thin Films, M.sc. Thesis, McMaster University, 2017

10. S.N. Fernandes, P.L. Almeida, N. Monge, L.E. Aguirre, D. Reis, C.L.P. de Oliveira, A.M.F. Neto, P. Pieranski, M.H. Godinho, Adv. Mater. 29, 1603560 (2017)

11. S.N. Fernandes, Y. Geng, S. Vignolini, B.J. Glover, A.C. Trindade, J.P. Canejo, P.L. Almeida, P. Brogueira, M.H. Godinho, Macromol. Chem. Phys. 214, 25 (2012)

12. J.O. Fossum, Physica A 270, 270 (1999)

13. H. Kalo, M.W. Möller, M. Ziadeh, D. Dolejš, J. Breu, Appl. Clay Sci. 48, 39 (2010)

14. H. Kalo, W. Milius, J. Breu, RSC. Adv. 2, 8452 (2012) 
15. S. Farrokhpay, B. Ndlovu, D. Bradshaw, Miner. Eng. 96-97, 59 (2016)

16. M. Stoter, D.A. Kunz, M. Schmidt, D. Hirsemann, H. Kalo, B. Putz, J. Senker, J. Breu Langmuir 29, 1280 (2013)

17. M. Stöter, S. Rosenfeldt, J. Breu, Ann. Rev. Mater. Res. 45, 129 (2015)

18. E.L. Hansen, H. Hemmen, D.M. Fonseca, C. Coutant, K.D. Knudsen, T.S. Plivelic, D. Bonn, J.O. Fossum, Sci. Rep. 2, 618 (2012)

19. V. Nicolosi, M. Chhowalla, M.G. Kanatzidis, M.S. Strano, J.N. Coleman, Science 340, $72(2013)$

20. E. DiMasi, J.O. Fossum, T. Gog, C. Venkataraman, Phys. Rev. E 64, 061704 (2001)

21. D.M. Fonseca, Y. Méheust, J.O. Fossum, K.D. Knudsen, K.P.S. Parmar, Phys. Rev. E 79, $021402(2009)$

22. H. Hemmen, N.I. Ringdal, E.N. De Azevedo, M. Engelsberg, E.L. Hansen, Y. Méheust, J.O. Fossum, K.D. Knudsen, Langmuir 25, 12507 (2009)

23. A. Liu, A. Walther, O. Ikkala, L. Belova, L.A. Berglund, Biomacromolecules 12, 633 (2011)

24. J.F. Revol, H. Bradford, J. Giasson, R.H. Marchessault, D.G. Gray, Int. J. Biol. Macromol. 14, 170 (1992)

25. Y. Mao, K. Liu, C. Zhan, L. Geng, B. Chu, B.S. Hsiao, Phys. Chem. B 121, 1340 (2017)

26. M.G. Aguayo, A.F. Pérez, G. Reyes, C. Oviedo, W. Gacitúa, R. Gonzalez, O. Uyarte, Polymers 10, 1145 (2018)

27. I.K.I. Al-khateeb, S.M. Hussin, Y.M. Al-Obaidi, Int. J. Mater. Chem. Phys. 1, 99 (2015)

28. D. Gaspar, S.N. Fernandes, A.G. de Oliveira, J.G. Fernandes, P. Grey, R.V. Pontes, L. Pereira, R. Martins, M.H. Godinho, E. Fortunato, Nanotechnology 25, 094008 (2014)

29. I. Carrillo, R.T. Mendonça, M. Ago, O.J. Rojas, Cellulose 25, 1011 (2018)

30. D. Klemm, B. Heublein, H.P. Fink, A. Bohn, Angew. Chemie - Int. Ed. 44, 3358 (2005)

31. L. Segal, J.J. Creely, A.E. Martin, C.M. Conrad, Textile Res. J. 29, 786 (1959)

32. I. Hoeger, O.J. Rojas, K. Efimenko, O.D. Velev, S.S. Kelley, Soft Matter, 7, 1957 (2011)

33. N.F. Vasconcelos, J.P.A. Feitosa, F.M.P. Gama, J.P.S. Morais, F.K. Andrade, M.S.M. de Souza Filho, M.F. Rosa, Carbohydr. Polym. 155, 425 (2017)

34. M. Roman, W.T. Winter, Biomacromolecules 5, 1671 (2004)

35. M.A. Mohamed, W.N.W. Salleh, J. Jaafar, S.E.A.M. Asri, A.F. Ismail, RSC Adv. 5, $29842(2015)$

36. S. Elazzouzi-Hafraoui, Y. Nishiyama, J.L. Putaux, L. Heux, F. Dubreuil, C. Rochas, Biomacromolecules 9, 57 (2008)

37. W.Y. Hamad, T.Q. Hu, Can. J. Chem. Eng. 88, 392 (2010)

38. K.J. De France, K.G. Yager, T. Hoare, E.D. Cranston, Langmuir 32, 7564 (2016)

39. B. Frka-Petesic, G. Guidetti, G. Kamita, S. Vignolini, Adv. Mater. 29, 1701469 (2017)

40. J.B. Hayter, J. Penfold, Mol. Phys. 42, 109 (1981) 